\title{
Mussel-derived compound enhances dental filling durability
}

Researchers from the Faculty of Dentistry, the University of Hong Kong (HKU),

Wuhan University (WHU) and the Peking University Shenzhen Hospital have found that a compound found in mussels helps increase the durability of a dental filling.

In a journal article published in Materials Today Bio entitled 'Enhancing resin-dentin bond durability using a novel mussel-inspired monomer,' ${ }^{1}$ the researchers explain why this is a promising clinical finding for the future of dental filling treatments.

The durability of a dental filling highly depends on the longevity and stability of the bond between the compound (resin) and the hard tissue of the tooth (dentine). This is where mussels play a role.

Small shellfish widespread in the marine environment, mussels offer unique wet adhesion properties which have long been of interest to the scientific community. Thus, the interaction between mussel plaques and substrates under humid environments has been extensively studied for insights on potential clinical applications. The study revealed that a compound found in an adhesive protein in mussels could strengthen the resin-dentine bond.

Professor Cynthia Kar Yung Yiu, Clinical Professor in Paediatric Dentistry, HKU who is leading the research team, said: 'Mussels need to maintain their adhesiveness under harsh marine environments, including humidity, drastic change of water temperature and $\mathrm{pH}$ value, sudden shocks and so on. These are similar to the daily activities that happen in the oral cavity. Our research aimed to understand the adhesive properties of the compounds from mussels, which may improve the durability and longevity of dental fillings'.

The durability of the bond between resin and dentine can be affected by several factors, such as the humidity inside the oral cavity and repeated mechanical stress induced by chewing. Therefore, it remains a clinically significant challenge for the dentist as well as the patient as it leads to

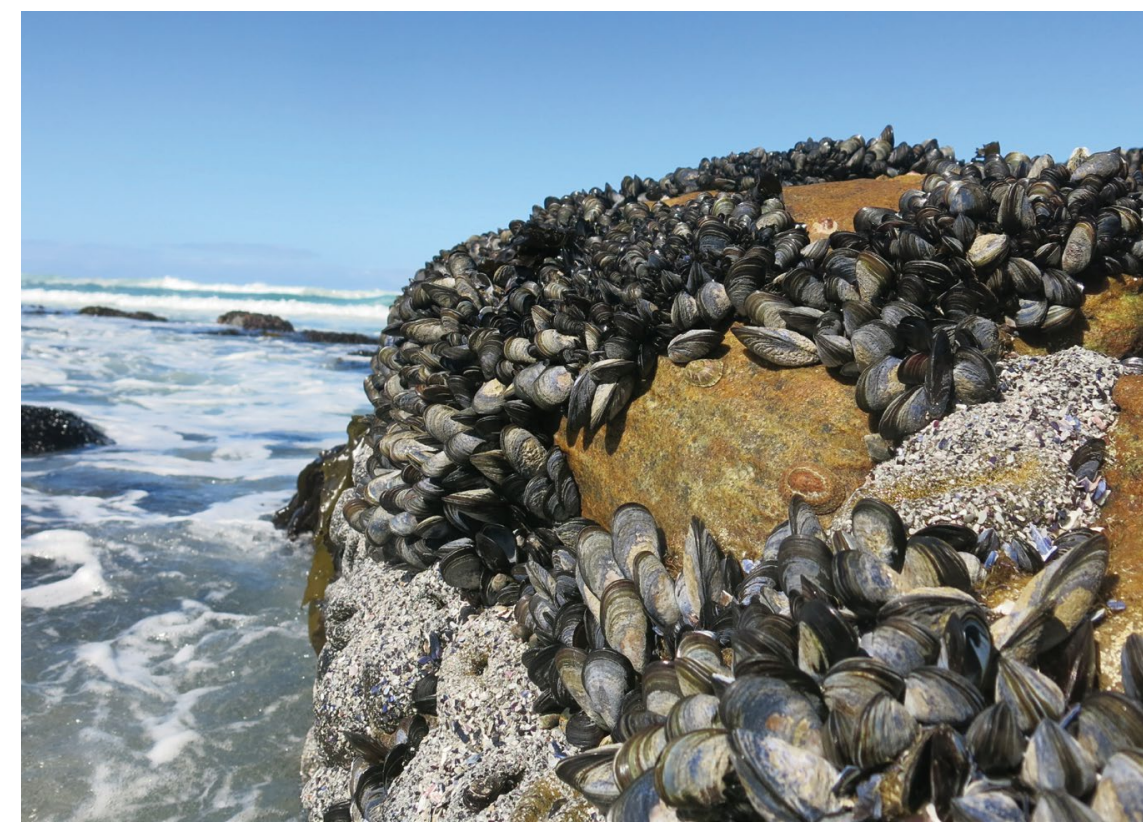

frequent replacement of the dental fillings at extra costs.

The study revealed that the wet adhesive property of mussels is attributed to the amino acid Dopa which they secrete. Based on the result, the team successfully applied $\mathrm{N}$-(3,4-dihydroxyphenethyl)methacrylamide (DMA), a mussel-derived compound, as a dental adhesive. The team further tested the durability of this resin-dentine interface versus the new DMA bond.

The control group and those with distinct concentrations of DMA underwent different tests including thermocycling ageing, a process where dental materials are exposed to varied temperatures. The international standard for testing dental adhesives requires test specimens to be held repeatedly first in $5{ }^{\circ} \mathrm{C}$ cold water and then in $55^{\circ} \mathrm{C}$ hot water for a large number of cycles. The results after subsequent testing invariably show a decrease in adhesive strength.

The researchers then employed the nanoleakage evaluation method in which an acid is added to measure the quality of the bond. The team used a silver nitrate solution to observe the patterns of nanoleakage.

In the resin-dentine interface, the thermocycling ageing process caused the formation of cracks and fissures which further led silver particles to infiltrate and settle along the bonded interface. The silver deposition therefore clearly reflected the water-filled and destructed areas along the interface. In the control group, silver particles were observed spreading along the resin-dentine interface and infiltrating inside dentinal tubules after ageing (nanoleakage deposition increased from $36.57 \%$ to $50.41 \%$ ). On the contrary, no obvious change could be detected for the DMA-treated groups (nanoleakage deposition around 20\%). The team thus deduced that DMA could strengthen the resin-dentine bond and its durability and is believed to increase the longevity of a dental filling.

Co-author Dr James Kit Hon Tsoi said: 'This research discovered that DMA is effective in strengthening the resin-dentine bond and improves its durability. The cytotoxicity is also similar to the resin monomers in traditional dental adhesives. It is believed that this compound may be commercialised in the future'.

\section{Reference}

1. Li K, Yao C, Sun Y et al. Enhancing resin-dentin bond durability using a novel mussel-inspired monomer. Mater Today Bio 2021; doi: 10.1016/j. mtbio.2021.100174. 